\title{
Analysis of heart rate control to assess thermal sensitivity responses in Brazilian toads
}

\author{
J.E.S. Natali, B.T. Santos, V.H. Rodrigues and J.G. Chauí-Berlinck \\ Departamento de Fisiologia, Instituto de Biociências, Universidade de São Paulo, São Paulo, SP, Brasil
}

\begin{abstract}
In anurans, changes in ambient temperature influence body temperature and, therefore, energy consumption. These changes ultimately affect energy supply and, consequently, heart rate (HR). Typically, anurans living in different thermal environments have different thermal sensitivities, and these cannot be distinguished by changes in HR. We hypothesized that Rhinella jimi (a toad from a xeric environment that lives in a wide range of temperatures) would have a lower thermal sensitivity regarding cardiac control than $R$. icterica (originally from a tropical forest environment with a more restricted range of ambient temperatures). Thermal sensitivity was assessed by comparing animals housed at $15^{\circ}$ and $25^{\circ} \mathrm{C}$. Cardiac control was estimated by heart rate variability (HRV) and heart rate complexity (HRC). Differences in HRV between the two temperatures were not significant $(\mathrm{P}=0.214$ for $R$. icterica and $\mathrm{P}=0.328$ for $R$. jimi), whereas HRC differences were. All specimens but one $R$. jimi had a lower $\mathrm{HRC}$ at $15^{\circ} \mathrm{C}$ (all $\mathrm{P}<0.01$ ). These results indicate that $R$. jimi has a lower thermal sensitivity and that cardiac control is not completely dependent on the thermal environment because HRC was not consistently different between temperatures in all $R$. jimi specimens. This result indicates a lack of evolutive trade-offs among temperatures given that heart rate control at $25^{\circ} \mathrm{C}$ is potentially not a constraint to heart rate control at $15^{\circ} \mathrm{C}$.
\end{abstract}

Key words: Anuran; Cardiac control; Heart rate complexity; Heart rate variability; Thermal physiology

\section{Introduction}

Animals regulate or change their body temperature in response to ambient temperature, and changes in energy demands accompany this process. As a general rule, maintaining euthermia in the face of decreased ambient temperature requires an increase in metabolic rate by endotherms-homeotherms while ectotherms-heterotherms would experience a decrease in their metabolic rate when ambient temperature decreases.

These alterations in metabolic rate ultimately reflect changes at the cellular level, with various organs and systems related to the delivery of necessary metabolic substrates conforming to the metabolic variations. This delivery system is composed of many coupled systems, including gas exchange organs, circulation, and subcellular organelles such as mitochondria. Therefore, the heart has a very important role in the process of thermal adjustment because body temperature and metabolic rate are somehow kept in pace in organisms.

Changes in heart rate (HR) are related to the balance between sympathetic and parasympathetic excitation, which modulates HR by modifying the heart's pacemaker frequency and myocardial contraction strength (1). When measured by the length of beat-to-beat intervals, heart rate fluctuations are considered an indirect measure of autonomous cardiac control (1). Also, a decrease in this variable is correlated with several human cardiovascular diseases (1).

These control mechanisms and their implications are not as well studied in anurans as they are in mammals, but they seem to be basically the same in both groups. Some particularities do exist, such as no reflex hypotension induced by baroreceptors in Xenopus (2) and an absence of drug-induced autonomous tonus changes in Lithobates catesbeianus (3). At the same time, studies have shown that the heart of Rhinella schneideri is innervated by both sympathetic and parasympathetic systems (4). R. marina presents sympathetic tachycardia (5). In L. catesbeianus, epinephrine concentration increases in response to hemorrhage (3). This balance between sympathetic and parasympathetic systems in anurans demonstrates the relevance of studying $\mathrm{HR}$ changes in these organisms.

It is known that low temperatures can completely block vagal nerve activity in L. pipiens (6). Consequently, because

Correspondence: J.E.S. Natali <jesnatali@gmail.com>

Received March 16, 2014. Accepted September 2, 2014. First published online October 24, 2014. 
anurans often face wide temperature variation in their habitats and present mechanisms of HR control similar to mammals, it is relevant to quantitatively investigate cardiac control as a means of adaptation to thermal stress. The significance of such a marker (i.e., cardiac control) becomes even more clear considering that HRs occurring at different temperatures are unreliable to distinguish between two Bufonidae species with putatively different thermal sensitivities (i.e., HR does not seem to be linearly or similarly related to ambient temperature) (7).

Thermal sensitivity is perhaps the most important ecophysiological variable affecting ectotherm performance (8). However, even for the most studied physiological aspect (i.e., locomotor performance), the general understanding is considered poor (8). The basic goal of thermal sensitivity analysis is to observe a given performance as a function of body temperature. Even though this is typically carried out by measuring specific characteristics (e.g., thermal optimum, critical thermal limits, and $80 \%$ performance breadth) (8), it is reasonable to assume that species with dissimilar performance functions would perform differently at fixed temperatures. Moreover, because locomotor performance (therefore, an energy demanding system) is usually adjusted to ecologically relevant ranges of temperature in anurans (9), species living in different thermal environments might exhibit different HR control. The presence of these differences would advocate for the labile view of the evolution of thermal sensitivity. That is, physiological performance can vary greatly within and among species, which is in contrast to the conservative view that states that thermal physiology does not evolve readily (both viewpoints are reviewed in Ref. 8).

The present study assessed the disparity in cardiac variability/complexity of two different Bufonidae species when the animals were exposed to different ambient temperatures. The Bufonidae are a family of neobatrachian frogs with nearly cosmopolitan distribution (10). The studied species belong to the $R$. marina group and are phylogenetically close to each other. The group contains 10 species distributed throughout different habitats from Texas to Uruguay (11). R. jimi (Stevaux, 2002) are originally from xeric environments (Brazilian Caatinga region) and face temperatures from $20^{\circ} \mathrm{C}$ to more than $40^{\circ} \mathrm{C}$. On the other hand, $R$. icterica (Spix, 1824) originated in tropical forests (Brazilian Mata Atlântica region) and are exposed to a temperature variation from $15^{\circ}$ to $25^{\circ} \mathrm{C}$. Because of the wider range of temperatures in the Brazilian Caatinga, we hypothesized that $R$. jimi would have a lower thermal sensitivity than $R$. icterica. To our knowledge, this is the first study of thermal sensitivity from the perspective of HR control.

\section{Material and Methods}

Six R. jimi specimens $(161.7 \pm 10.3 \mathrm{~g})$ were fieldcollected in the Brazilian Caatinga (Angicos, Rio Grande do Norte), and five specimens of $R$. icterica $(120.3 \pm 7.1 \mathrm{~g}$ ) were collected in an area of tropical forest located in the city of São Paulo. All specimens were male. The animals were kept in a terrarium with food and water ad libitum for at least 1 month before the experiments. During this period, the animals were exposed to temperature ranges that resembled their natural environment and maintained in a 12:12-h light:dark cycle. The experiments were carried out during the winter. The animals were handled in accordance with the Brazilian rules at the time of the experiments (2006) and the protocol was approved by the Comissão de Ética no Uso de Animais-Instituto de Biociências (CEUAIB) of Universidade de São Paulo.

For each part of the experiment, a single specimen was randomly selected and taken to another terrarium with a 12:12-h light:dark cycle, given ad libitum water but no food, and kept there for $24 \mathrm{~h}$ at $25^{\circ} \mathrm{C}$. After that time, the animal was transferred to a plastic container where, after $1 \mathrm{~h}$ without any further manipulation, electrocardiogram (ECG) data were recorded for $3-4 \mathrm{~h}$ as described below. The animals were then kept at $15^{\circ} \mathrm{C}$ for another $24 \mathrm{~h}$. After this second period and at least $1 \mathrm{~h}$ after any handling, another ECG recording was obtained, and the animals were returned to the original terrarium. All ECG data were collected at a relative humidity of $70-80 \%$ and, to avoid chronobiological differences, at approximately the same time of the day.

ECG data were obtained using two superficial electrodes (Unilect Electrodes, Maersk Medical Ltd., Denmark) with a dorsoventral alignment. To improve data quality, a conductive gel was utilized (Eletrocardio-Gel, Multi Gel Ltda., Brazil). ECGs were recorded and digitalized using a sampling rate of $200 \mathrm{~Hz}$, an MP30-interface (Biopac Systems Inc., USA) and the Biopac Student Lab Pro software (Biopac Systems Inc.). Data obtained from one $R$. icterica was discarded because of problems in data acquisition (i.e., continuous motion of the animal, loss of electrode contact with the skin, and input noise from unknown sources). The ECG records obtained from one $R$. icterica and two $R$. jimi at $15^{\circ} \mathrm{C}$ were also discarded for similar reasons. Therefore, the ECG data that were evaluated came from three $R$. icterica and four $R$. jimi at $15^{\circ} \mathrm{C}$ and four $R$. icterica and six $R$. jimi at $25^{\circ} \mathrm{C}$.

$\mathrm{RR}$ intervals (the period between two $\mathrm{R}$ waves in an ECG) were extracted from raw ECG data using Matlab Suite (Matlab version 7.10.0.499, The MathWorks Inc., USA) using a set of implemented scripts. From the total vector of RR intervals, excerpts of 300 uninterrupted points (an appropriate number of points according to preliminary tests, resulting in 1 to 5 segments per animal per temperature) were used for the following procedures.

Cardiac control was assessed through frequency domain (heart rate variability, HRV) and a nonlinear method (heart rate complexity, HRC). HRV was estimated using a fast Fourier transform based on Welch's periodogram (window width: $256 \mathrm{~s}$ with $50 \%$ overlap) on each of the 
300-point RR vectors using Kubios HRV 2.1 (Biomedical Signal Analysis and Medical Imaging Group, University of Eastern Finland, Finland). Due to the lack of information for anurans, and as is usually done for nonhumans (e.g., 12), we used the classic frequency ranges as a basis for the spectral component analysis.

In this sense, for experiments at $25^{\circ} \mathrm{C}$, we employed $0.04-0.15 \mathrm{~Hz}$ for the low-frequency range (LF, related to both sympathetic and parasympathetic activity) and $0.15-$ $0.4 \mathrm{~Hz}$ for the high-frequency range (HF, associated with parasympathetic activity) (1). In light of the observed relation between $\mathrm{HR}$ at the two experimental temperatures (shown below) and published data $(13,14)$, for the HRV analysis at $15^{\circ} \mathrm{C}$, we considered a thermal sensitivity of 2 and, therefore frequencies of $0.02-0.075 \mathrm{~Hz}$ for the LF range and $0.075-0.2 \mathrm{~Hz}$ for the $\mathrm{HF}$ range. Because $\mathrm{HF}$ is associated with only one of the branches of the autonomous nervous system (see above) and also directly and positively correlated to HRV (1), we used the former variable to characterize the latter.

HRC is a promising tool for HR analysis (1) because nonlinear phenomena are certainly involved in the genesis of HR control (1). Moreover, since no a priori knowledge about the species under study is required (e.g., range of the spectral components), this method is suitable for exploratory studies in nonhumans.

HRC was estimated using approximate entropy (ApEn) (15), a nonlinear method employed to quantify the degree of order in time series. To remove the bias associated with choosing the window of comparison $(m)$ and the tolerance $(r)$ of the ApEn estimate, the present study made use of the strategy proposed by Lu et al. (16) and utilized the maximum ApEn for $m=1$. For simplicity, this "maximum ApEn" will still be referred to as ApEn.

The ApEn algorithm was applied to the same data series used in the HRV analysis. To increase the robustness of the analysis, as proposed in (17), a moving ApEn was performed in each of the 300-point vectors using a window of 150 points, resulting in 150 ApEn values $\left(A p E n_{v e c}\right)$ for each segment.

The HRV analysis considered the normalized HF power for each of the 300 point segments or, in case of multiple segments per specimen, the mean of those segments. The values obtained from each individual toad at $15^{\circ}$ and $25^{\circ} \mathrm{C}$ were compared using a paired Student's $t$-test.

In contrast to HRV, HRC analysis utilized the $A p E n_{\text {vec }}$ (see above); therefore, multiple values were obtained even in specimens where only one 300-point segment was available. Hence, differences in HRC at the two different temperatures were compared using a Student $t$-test for each specimen. All statistical tests were performed with Matlab built-in scripts, and differences were considered to be significant at $\mathrm{P} \leq 0.05$.

\section{Results}

Table 1 summarizes the normalized HF of the fast Fourier transform (nu HF), the ApEn and HR results

Table 1. Heart rate, ApEn, and nu HF obtained for the number (n) of segments for 4 Rhinella jimi and 6 Rhinella icterica at $15^{\circ}$ and $25^{\circ} \mathrm{C}$.

\begin{tabular}{|c|c|c|c|c|c|}
\hline Specimen & $\mathrm{n}$ & Temperature $\left({ }^{\circ} \mathrm{C}\right)$ & Hear rate $(\mathrm{bpm})$ & nu HF & ApEn \\
\hline \multicolumn{6}{|c|}{ Rhinella icterica } \\
\hline \multirow[t]{2}{*}{1} & 3 & 15 & $39.2 \pm 0.71$ & $20.97 \pm 10.8$ & $1.18 \pm 0.08^{*}$ \\
\hline & 3 & 25 & $70.5 \pm 2.16$ & $43.53 \pm 11.8$ & $1.59 \pm 0.08$ \\
\hline \multirow[t]{2}{*}{2} & 2 & 15 & $24.5 \pm 0.82$ & $1.64 \pm 0.67$ & $1.14 \pm 0.07^{*}$ \\
\hline & 3 & 25 & $36.1 \pm 2.40$ & $4.72 \pm 2.85$ & $1.40 \pm 0.07$ \\
\hline \multirow[t]{2}{*}{3} & 1 & 15 & $30.0 \pm 1.24$ & 1.78 & $1.08 \pm 0.05^{*}$ \\
\hline & 1 & 25 & $51.4 \pm 1.11$ & 8.45 & $1.34 \pm 0.05$ \\
\hline 4 & 2 & 25 & $46.5 \pm 2.55$ & $5.83 \pm 0.84$ & $1.25 \pm 0.13$ \\
\hline \multicolumn{6}{|c|}{ Rhinella jimi } \\
\hline \multirow[t]{2}{*}{1} & 2 & 15 & $21.1 \pm 2.01$ & $2.90 \pm 1.48$ & $1.36 \pm 0.09^{*}$ \\
\hline & 3 & 25 & $24.4 \pm 2.19$ & $15.94 \pm 7.73$ & $1.59 \pm 0.09$ \\
\hline \multirow[t]{2}{*}{2} & 4 & 15 & $14.6 \pm 5.09$ & $6.65 \pm 6.83$ & $1.63 \pm 0.15$ \\
\hline & 5 & 25 & $24.6 \pm 6.72$ & $13.48 \pm 10.74$ & $1.61 \pm 0.16^{*}$ \\
\hline \multirow[t]{2}{*}{3} & 2 & 15 & $24.8 \pm 7.31$ & $3.28 \pm 2.13$ & $1.33 \pm 0.15^{*}$ \\
\hline & 2 & 25 & $24.8 \pm 2.01$ & $22.55 \pm 0.2$ & $1.64 \pm 0.04$ \\
\hline 4 & 2 & 25 & $33.2 \pm 2.02$ & $13.18 \pm 4.12$ & $1.58 \pm 0.08$ \\
\hline 5 & 4 & 25 & $63.6 \pm 2.10$ & $8.37 \pm 5.79$ & $1.35 \pm 0.08$ \\
\hline \multirow[t]{2}{*}{6} & 4 & 15 & $16.0 \pm 5.32$ & $14.03 \pm 13.13$ & $1.56 \pm 0.1^{*}$ \\
\hline & 3 & 25 & $24.0 \pm 4.76$ & $4.15 \pm 1.34$ & $1.66 \pm 0.21$ \\
\hline
\end{tabular}

Data are reported as means \pm SD, except when heart rate variability was obtained in only one segment. ApEn: approximate entropy; nu HF: normalized high frequency power; bpm: beats per minute; * Significant difference between temperatures ( $\mathrm{P}<0.01$, Student $t$-test). 


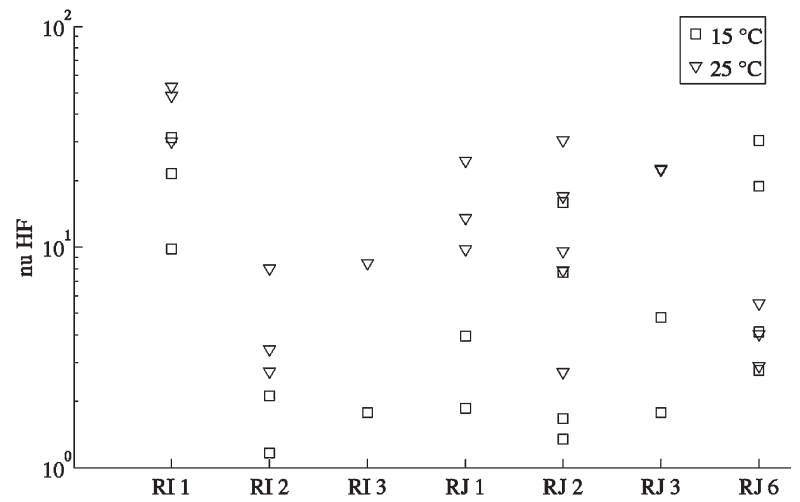

Figure 1. High frequency power of heart rate variability analysis for each specimen at both temperatures. Normalized high frequency power (nu HF, in log scale) obtained through power spectrum density analysis for 3 Rhinella icterica (RI 1-3) and 4 Rhinella jimi (RJ 1-3 and 6). Squares denote that the data were obtained at $15^{\circ} \mathrm{C}$ whereas triangles indicate results at $25^{\circ} \mathrm{C}$.

observed for all $R$. icterica and $R$. jimi study specimens. A decrease of $10^{\circ} \mathrm{C}$ resulted in nearly a 1.5 -times reduction in HR.

Even though it was possible to detect a decrease of $\mathrm{HRV}$ at $15^{\circ} \mathrm{C}$ in both species (as shown in Figure 1), these differences were not significant $(P=0.214$ for $R$. icterica and $\mathrm{P}=0.328$ for $R$. jimi). Furthermore, nu HF was higher at $25^{\circ} \mathrm{C}$ in only one $R$. jimi specimen (number 6).

$\mathrm{HRC}$ was significantly lower at $15^{\circ} \mathrm{C}$ in all $R$. icterica (all $\mathrm{P}<0.001$ ) but not in all $R$. jimi (specimen number 2 had a significantly higher $\mathrm{HRC}$ at $\left.15^{\circ} \mathrm{C}, \mathrm{P}<0.001\right)$. This is additional evidence that it is possible for $R$. jimi to have higher cardiac control at a lower temperature, therefore suggesting that this species is less sensitive to changes in temperature than $R$. icterica. Figure 2 illustrates this difference in sensitivity between the species.

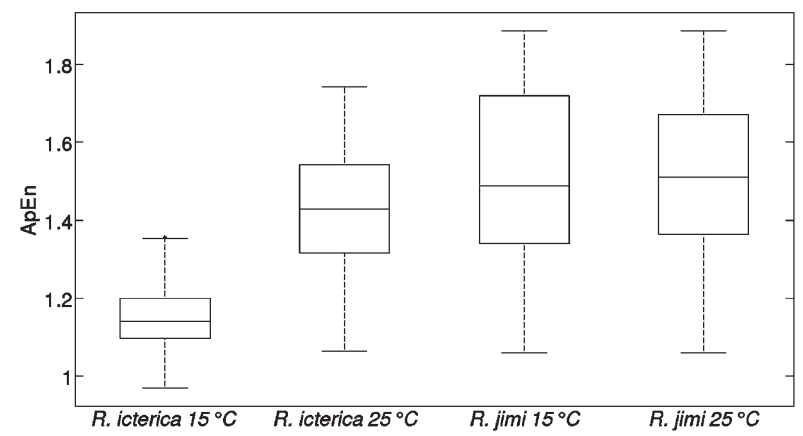

Figure 2. Approximate entropy (ApEn) values for Rhinella icterica and Rhinella jimi at $15^{\circ}$ and $25^{\circ} \mathrm{C}$. The middle line is the median, the top and bottom edges of the box are the 25th and 75th percentiles, and the bars indicate the maximum and minimum data points.

\section{Discussion}

This study was designed to discover the previously overlooked effect of temperature on $\mathrm{HR}$ control in anurans. To accomplish this, $\mathrm{HRV} / \mathrm{HRC}$ at $15^{\circ}$ and $25^{\circ} \mathrm{C}$ were measured in two species from different environments and compared. The results showed that HRV is not sufficient to differentiate the two thermal states and consequently did not distinguish these two species in terms of their thermal responses. On the other hand, HRC successfully discriminated these aspects. To our knowledge, this is the first study to use these analytical tools to investigate cardiac control in anurans.

$\mathrm{HRC}$ was lower at $15^{\circ} \mathrm{C}$ in both $R$. icterica and $R$. jimi (except for one $R$. jimi specimen). This result can be explained by the effect of the decreased temperature on the parasympathetic nervous system (6). However, the reason why one of the toads had a higher HRC at a lower temperature is still unclear. Nevertheless, this result highlights the importance and potential use of HRC as an indicator of thermal sensitivity. Moreover, the differences in cardiac control between these species of the same genus point toward the labile view of the evolution of thermal physiology (i.e., a more rapid evolution in response to the environment). However, studies in other groups are necessary to identify whether this is a general pattern or a taxa-dependent factor.

In agreement with our hypothesis, $R$. jimi had a lower thermal sensitivity than $R$. icterica, which is consistent with a lack of evolutionary trade-offs between HR control at high and low temperatures in Caatinga species. This means that, assuming a common curve of relative performance $v s$ body temperature (didactically reviewed in 8), $R$. icterica HR control at $25^{\circ} \mathrm{C}$ was potentially not a constraint to HR control at $15^{\circ} \mathrm{C}$ (i.e., there was an increase in the breadth of the performance curve instead of a shift). The lack of evolutionary trade-offs between temperatures is a feature shared with numerous other species (refer to Ref. 8 for examples). R. jimi is a recently described species (18), so its thermal physiology and natural history are poorly studied. Nevertheless, data from a coexistent species ( $R$. granulosas) (19) suggest that $R$. jimi do not experience temperatures lower than $20^{\circ} \mathrm{C}$. Therefore, this low thermal sensitivity is sustained even at a temperature uncharacteristic to the species.

$R$. icterica, on the other hand, is a species that reproduces in winter $(20)$ and is likely to be active at $15^{\circ} \mathrm{C}$, as in our experimental conditions. Therefore, since a decrease of HRC was found at this temperature for all $R$. icterica, it is possible that, for the studied species, a higher level of cardiac control is more dependent on the range of environmental temperatures to which the animals are exposed (higher in $R$. jimi, see Introduction) than on usual exposure to a specific temperature (lower in R. icterica). In relation to locomotor performance, this pattern of temperature sensitivity is different from that typically seen in anurans 
(reviewed in Refs. 8,9). However, exceptions do exist, and unusual situations where the field body temperature does not match either the preferred body temperature or the temperature that maximizes locomotor and calling performance have been described $(8,9)$.

Finally, it is necessary to point out an important limitation of the present work, namely the small sample size. Because of this, it is possible that some results, for instance the nonsignificant difference of HRV between temperatures, are a consequence of the small sample size and not of the method itself. Therefore, it is not possible to discard the use of HRV to investigate thermal sensitivity in anurans.

Despite this shortcoming, this is the first time that HR control was used to investigate thermal sensitivity in anurans, and we believe that this study may serve as an inspiration for future work in this area and to contribute to the discussion of anuran thermal physiology.

\section{References}

1. Task Force of the European Society of Cardiology and the North American Society of Pacing and Electrophysiology. Heart rate variability. Standards of measurement, physiological interpretation, and clinical use. Eur Heart J 1996; 17: 354-381, doi: 10.1093/oxfordjournals.eurheartj.a014868.

2. Bernardi M, Deslauriers R, Docherty J, Rossi C, Rossini L, Rossini $P$, et al. Spectral analysis of intercycle heart fluctuations in Xenopus laevis, conscious or spinalized, treated with calcium channel blockers. Part I. Gen Pharmacol 1997; 29: 477-481, doi: 10.1016/S0306-3623(96)00462-4.

3. Burggren W, Doyle M. The action of acetylcholine upon heart rate changes markedly with development in bullfrogs. J Exp Zool 1986; 240: 137-140, doi: 10.1002/jez.1402400117.

4. Hoffmann A, Romero SM. Effect of the dry-cold season dormancy on the tonic and phasic neural control of heart rate in the toad, Bufo paracnemis. J Exp Zool 2000; 287: 15-20, doi: 10.1002/1097-010X(20000615)287:1<15::AID-JEZ2>3.0.CO;2-E.

5. Bramich NJ, Edwards FR, Hirst GD. Sympathetic nerve stimulation and applied transmitters on the sinus venosus of the toad. J Physiol 1990; 429: 349-375.

6. Lund GF, Dingle H. Seasonal temperature influence on vagal contol of diving bradycardia in the frog (Rana Pipiens). J Exp Biol 1968; 48: 265-277.

7. Chapovetsky V, Katz U. Effects of season and temperature acclimation on electrocardiogram and heart rate of toads. Comp Biochem Physiol A Mol Integr Physiol 2003; 134: 7783, doi: 10.1016/S1095-6433(02)00224-6.

8. Angilletta MJ, Niewiarowski PH, Navas CA. The evolution of thermal physiology in ectotherms. J Therm Biol 2002; 27 : 249-268, doi: 10.1016/S0306-4565(01)00094-8.

9. Navas CA, Gomes FR, Carvalho JE. Thermal relationships and exercise physiology in anuran amphibians: integration and evolutionary implications. Comp Biochem Physiol Part A: Mol Integr Physiol 2008; 151: 344-362, doi: 10.1016/j.cbpa.2007. 07.003.

10. Pramuk JB. Phylogeny of South American Bufo (Anura: Bufonidae) inferred from combined evidence. Zool J Linn Soc 2006; 146: 407-452, doi: 10.1111/j.1096-3642.2006.00212.x.

11. Maciel NM, Collevatti RG, Colli GR, Schwartz EF. Late
To summarize, the results demonstrated a species difference in the HR control response at different temperatures; therefore, this physiological aspect warrants further study in the context of thermal sensitivity. Data from additional groups are required to achieve greater understanding of HR control in the evolution of thermal sensitivity. The results showed that the analysis of HRC through ApEn was able to detect thermal differences between species; therefore, this seems to be a powerful and reliable tool to approach physiological systems in anurans and other species.

\section{Acknowledgments}

The authors would like to thank Carlos Jared for providing the specimens. Research supported by FAPESP (\#06/0212-0) to J.G. Chauí-Berlinck.

Miocene diversification and phylogenetic relationships of the huge toads in the Rhinella marina (Linnaeus, 1758) species group (Anura: Bufonidae). Mol Phylogenet Evol 2010; 57: 787-797, doi: 10.1016/j.ympev.2010.08.025.

12. Yeragani VK, Collins HL, Rao KAR, Rodenbaugh DW, DiCarlo SE. Decreased chaos after exercise in cardiac output time series of rats: a preliminary report. Nonlinear Anal Real World Appl 2003; 4: 307-316, doi: 10.1016/S1468-1218(02)00023-8.

13. Carey $\mathrm{C}$. Effect of constant and fluctuating temperatures on resting and active oxygen consumption of toads, Bufo boreas. Oecologia 1979; 39: 201-212, doi: 10.1007/BF00348069.

14. Putnam RW, Bennett AF. Thermal dependence of behavioural performance of anuran amphibians. Anim Behav 1981; 29: 502-509, doi: 10.1016/S0003-3472(81)80111-X.

15. Pincus SM. Approximate entropy as a measure of system complexity. Proc Natl Acad Sci U S A 1991; 88: 2297-2301, doi: 10.1073/pnas.88.6.2297.

16. Lu S, Chen X, Kanters JK, Solomon IC, Chon KH. Automatic selection of the threshold value $\mathrm{R}$ for approximate entropy. IEEE Trans Biomed Eng 2008; 55: 19661972, doi: 10.1109/TBME.2008.919870.

17. Hu X, Miller C, Vespa P, Bergsneider M. Adaptive computation of approximate entropy and its application in integrative analysis of irregularity of heart rate variability and intracranial pressure signals. Med Eng Phys 2008; 30: 631-639, doi: 10.1016/j.medengphy.2007.07.002.

18. Stevaux M. A new species of Bufo Laurenti (Anura, Bufonidae) from Northeastern Brazil. Rev Bras Zool 2002; 19: 235-242, doi: 10.1590/S0101-81752002000500018.

19. Navas CA, Antoniazzi MM, Carvalho JE, Suzuki H, Jared C. Physiological basis for diurnal activity in dispersing juvenile Bufo granulosus in the Caatinga, a Brazilian semi-arid environment. Comp Biochem Physiol A Mol Integr Physiol 2007; 147: 647-657, doi: 10.1016/j.cbpa.2006.04.035.

20. Titon B Jr, Navas CA, Jim J, Gomes FR. Water balance and locomotor performance in three species of neotropical toads that differ in geographical distribution. Comp Biochem Physiol A Mol Integr Physiol 2010; 156: 129-135, doi: 10.1016/ j.cbpa.2010.01.009. 